\title{
Invariance principle for diffusions in random environment
}

\author{
S.Struckmeier \\ Department of Mathematics, Universität Bielefeld, Universitätsstr. 25, 33615 Bielefeld, Germany
}

Received January 31, 2008

\begin{abstract}
We will show an invariance principle for the diffusive motion of a particle interacting with a random frozen configuration of infinitely many other particles in $\mathbb{R}^{d}$. The interaction is described by a symmetric, translation invariant pair potential with repulsion at zero distance and proper decay at infinity.
\end{abstract}

Key words: invariance principle, random environment, configuration spaces, diffusion with singular interaction

PACS: $02.50 . \mathrm{Ga}$

\section{Introduction}

In this article we are going to show an invariance principle, i.e. convergence of a process to Brownian motion under a space-time scaling, for the diffusive motion of a particle interacting with infinitely many other particles in $\mathbb{R}^{d}, d \geqslant 2$. To this end we will use a general approach developed by A. De Masi, P.A. Ferrari, S. Goldstein, and W.D. Wick [3] and C. Kipnis and S.R.S. Varadhan $[5]$.

The random environment we consider is a random configuration $\gamma$, i.e. a locally finite subset of $\mathbb{R}^{d}$, chosen with respect to a grand canonical Gibbs measure $\mu_{\mathrm{E}}$ with respect to a potential $V_{\mathrm{E}}$. A particle $x$ performs a diffusive motion $\left(X_{t}\right)_{t \geqslant 0}$ in $\mathbb{R}^{d} \backslash \gamma$ and interacts with the (frozen) points in $\gamma$ via a symmetric, translation invariant pair potential $V_{\mathrm{I}}$. De Masi et al. also discussed this situation in the case of a positive, compactly-supported $C^{\infty}$ interaction potential. Furthermore, they assumed $V_{\mathrm{I}}=V_{\mathrm{E}}$. We will assume that $V_{\mathrm{I}}$ has a singularity at the origin, that is repulsion of particles at distance zero, and a proper decay at infinity. But it has to be neither positive nor of finite range. The case that $V_{\mathrm{I}}$ is the Lennard-Jones potential is included. Furthermore, it does not have to be the same potential $V_{\mathrm{E}}$ to which the Gibbs measure corresponds. By using newer results (cf. $[7,10]$ ) on diffusions in random environment we can construct the corresponding stochastic process in this setting, which describes the motion of the particle in the random environment, and then apply the technique by De Masi et al. to obtain an invariance principle. The main idea of this approach is to write $\left(X_{t}\right)_{t \geqslant 0}$ as a functional of its environment process $\left(\xi_{t}\right)_{t \geqslant 0}$, i.e. the motion of the environment as seen from the particle. $\left(\xi_{t}\right)_{t \geqslant 0}$ is a process on the configuration space $\Gamma$. De Masi et al. formulated conditions on this environment process which imply an invariance principle for the original process $\left(X_{t}\right)_{t \geqslant 0}$.

\section{Configuration spaces}

Here we will briefly recall the framework of configuration space analysis. For a general overview we refer to e.g. $[1,2]$.

The configuration space $\Gamma:=\Gamma\left(\mathbb{R}^{d}\right)$ is defined as the set of all locally finite subsets of $\mathbb{R}^{d}$, i.e.

$$
\Gamma:=\left\{\gamma \subset \mathbb{R}^{d}:|\gamma \cap K|<\infty \text { for any } K \subset \mathbb{R}^{d} \text { compact }\right\} \text {. }
$$


Here $|\gamma \cap K|$ denotes the cardinality of the set $\gamma \cap K$. The sets $\gamma \in \Gamma$ are called configurations. We will think of a configuration $\gamma$ as a set of indistinguishable particles in $\mathbb{R}^{d}$. Every $\gamma \in \Gamma$ can be identified with a positive Radon measure on $\mathbb{R}^{d}$ via

$$
\gamma \equiv \sum_{y \in \gamma} \delta_{y}
$$

where $\delta_{y}$ denotes the Dirac measure with mass in $y \in \mathbb{R}^{d}$, and the empty configuration is identified with the zero measure. Then, as a subset of the set of all positive Radon measures, $\Gamma$ is naturally topologized by the (subspace topology induced by the) vague topology, i.e., the weakest topology on $\Gamma$ such that all maps

$$
\Gamma \ni \gamma \mapsto\langle f, \gamma\rangle:=\int_{\mathbb{R}^{d}} f(x) \mathrm{d} \gamma(x)=\sum_{y \in \gamma} f(y)
$$

are continuous, where $f \in C_{0}\left(\mathbb{R}^{d}\right)$, i.e., $f$ is a continuous function on $\mathbb{R}^{d}$ with compact support. Let $\mathcal{B}(\Gamma)$ denote the Borel $\sigma$-algebra on $\Gamma$ corresponding to this topology. In particular, $\gamma \mapsto\langle f, \gamma\rangle$ is $\mathcal{B}(\Gamma)$-measurable for all $f \in C_{0}\left(\mathbb{R}^{d}\right)$. For $B \in \mathcal{B}\left(\mathbb{R}^{d}\right)$ we define $N_{B}: \Gamma \rightarrow \mathbb{N}_{0} \cup\{+\infty\}$ by $N_{B}(\gamma):=\gamma(B)=|\gamma \cap B|$. Then

$$
\mathcal{B}(\Gamma)=\sigma\left(\left\{N_{\Lambda}: \Lambda \subset \mathbb{R}^{d} \text { open, pre-compact }\right\}\right) .
$$

Consider a pair potential $V$, i.e., a Lebesgue-measurable function $V: \mathbb{R}^{d} \rightarrow \mathbb{R} \cup\{+\infty\}$, such that $V(-x)=V(x)$ for any $x \in \mathbb{R}$. The relative energy of interaction between a particle at point $x \in \mathbb{R}^{d}$ and a configuration $\gamma \in \Gamma$ via the potential $V$ is defined as

$$
E^{V}(x, \gamma):= \begin{cases}\sum_{y \in \gamma} V(x-y), & \text { if } \sum_{y \in \gamma}|V(x-y)|<+\infty \\ +\infty, & \text { otherwise }\end{cases}
$$

A probability measure $\mu$ on $(\Gamma, \mathcal{B}(\Gamma))$ is called a (grand canonical) Gibbs measure with respect to activity parameter $z>0$ and potential $V$ if it satisfies the Georgii-Nguyen-Zessin identity (GNZ): for any positive $\mathcal{B}\left(\mathbb{R}^{d}\right) \times \mathcal{B}(\Gamma)$-measurable function $H$ it holds that

$$
\int_{\Gamma} \sum_{x \in \gamma} H(x, \gamma) \mathrm{d} \mu(\gamma)=\int_{\Gamma} \int_{\mathbb{R}^{d}} H(x, \gamma \cup x) \mathrm{e}^{-E^{V}(x, \gamma)} z \mathrm{~d} x \mathrm{~d} \mu(\gamma) .
$$

There exist equivalent definitions of Gibbs measures, e.g. via Dobrushin-Lanford-Ruelle approach. To this end, cf., e.g., [8].

A system of non-negative measurable symmetric functions $k_{\mu}^{(n)}$ on $\left(\mathbb{R}^{d}\right)^{n}$ is called the system of correlation functions of $\mu$ if for any non-negative measurable symmetric function $f^{(n)}$ on $\left(\mathbb{R}^{d}\right)^{n}$ we have that

$$
\begin{aligned}
\int_{\Gamma} \sum_{\left\{x_{1}, \ldots, x_{n}\right\} \subset \gamma} f^{(n)}\left(x_{1}, \ldots, x_{n}\right) \mathrm{d} \mu(\gamma) & \\
& =\frac{1}{n !} \int_{\left(\mathbb{R}^{d}\right)^{n}} f^{(n)}\left(x_{1}, \ldots, x_{n}\right) k_{\mu}^{(n)}\left(x_{1}, \ldots, x_{n}\right) \mathrm{d} x_{1} \cdots \mathrm{d} x_{n} .
\end{aligned}
$$

A constant $\xi>0$ is called Ruelle bound if it satisfies

$$
k_{\mu}^{(n)} \leqslant \xi^{n}, \quad n \in \mathbb{N}
$$

It is well-known that tempered Gibbs measures corresponding to superstable, lower regular, integrable (in the sense of (10)) pair potentials have correlation functions with Ruelle bound, cf. [11]. 


\section{Construction of the process}

We want to describe the diffusive motion of a particle $x \in \mathbb{R} \backslash \gamma$, where $\gamma \in \Gamma$ is a configuration randomly chosen with respect to some Gibbs measure. From now on assume that $d \geqslant 2$, then $\mathbb{R}^{d} \backslash \gamma$ is connected for any $\gamma$.

Consider a symmetric, translation invariant pair potential $V$, i.e., $V(x-y)=\tilde{V}(|x-y|)$, $x, y \in \mathbb{R}^{d}$, for some proper function $\tilde{V}:(0,+\infty) \rightarrow \mathbb{R}$. We assume repulsion at zero distance and decay of the potential at infinity, i.e.,

$$
\lim _{|x| \rightarrow 0} V(x)=+\infty \quad \text { and } \lim _{|x| \rightarrow+\infty} V(x)=0 .
$$

Assume that the relative energy $E^{V}(x, \gamma)$ is finite for every $x \in \mathbb{R}^{d} \backslash \gamma$. (This holds under the assumptions of Theorem 1 and Theorem 2 below.) Let

$$
\rho_{\gamma}(x):=\mathrm{e}^{-E^{V}(x, \gamma)},
$$

then

$$
\beta_{\gamma}(x):=\frac{\nabla \rho_{\gamma}(x)}{\rho_{\gamma}(x)}=-\sum_{y \in \gamma} \nabla V(x-y),
$$

the logarithmic derivative of $\rho_{\gamma}$, is well-defined Lebesgue-a.e. The motion of the particle in $\mathbb{R}^{d} \backslash \gamma$ should be described by the following stochastic differential equation (SDE):

$$
\left\{\begin{aligned}
\mathrm{d} X_{t} & =\beta_{\gamma}\left(X_{t}\right) \mathrm{d} t+\mathrm{d} W_{t}, \\
X_{0} & =x_{0}\left(\in \mathbb{R}^{d} \backslash \gamma\right) .
\end{aligned}\right.
$$

Here $W_{t}$ is a standard Brownian motion on $\mathbb{R}^{d}$.

Define the set

$$
\Gamma_{\mathrm{ad}}:=\left\{\gamma \in \Gamma: \forall r>0 \exists c=c(\gamma, r) \text { with }|\gamma \cap B(x, r)| \leqslant c \cdot \log (2+|x|) \forall x \in \mathbb{R}^{d}\right\}
$$

of admissible configurations. Here $B(x, r)$ denotes the ball with center $x$ and radius $r$. For many classes of probability measures $\nu$ on $\Gamma$ we have $\nu\left(\Gamma_{\mathrm{ad}}\right)=1$, in particular for Ruelle measures corresponding to superstable pair potentials, cf. [6,9].

The following result is due to N.V. Krylov and M. Röckner [10].

Theorem 1. Assume that the potential $V$ satisfies (4) and the following assumptions:

(i) $V \in C^{1}\left(\mathbb{R}^{d} \backslash\{0\}\right)$;

(ii) there exist constants $c>0, \alpha>d / 2, \varepsilon \in[1,2)$ such that

$$
\begin{array}{r}
|V(x)|+|\nabla V(x)| \leqslant c\left(1+|x|^{2}\right)^{-\alpha} \text { for }|x| \geqslant 1 ; \\
\Delta V(x) \leqslant c\left(\mathrm{e}^{\varepsilon(V(x)+U(x))}-1\right) \text { for }|x|>0
\end{array}
$$

in the sense of distributions on $\mathbb{R}^{d} \backslash\{0\}$, where $U(x):=c\left(1+|x|^{2}\right)^{-\alpha}$.

Let $\gamma \in \Gamma_{a d}$. Then (5) has a unique strong solution defined for all times if $x_{0} \in \mathbb{R}^{d} \backslash \gamma$.

Yu.G. Kondratiev, A.Yu. Konstantinov and M. Röckner [7] have discussed earlier the slightly different stochastic differential equation

$$
\left\{\begin{aligned}
\mathrm{d} X_{t} & =\beta_{\gamma}\left(X_{t}\right) \mathrm{d} t+\sqrt{2} \mathrm{~d} W_{t}, \\
X_{0} & =x_{0}\left(\in \mathbb{R}^{d} \backslash \gamma\right),
\end{aligned}\right.
$$

with positive definite diffusion operator $\left(H, C_{0}^{\infty}\left(\mathbb{R}^{d}\right)\right)$,

$$
H u=-\Delta u-\langle\beta, \nabla u\rangle,
$$

on $L^{2}\left(\mathbb{R}^{d}, \rho_{\gamma} \mathrm{d} x\right)$. They proved the following result: 
Theorem 2. Assume that $V$ satisfies the following conditions:

(i) $V \in C^{2}\left(\mathbb{R}^{d} \backslash\{0\}\right)$, and $V \geqslant-$ for some $a>0$;

(ii) $\exp \left(-\frac{1}{2} V\right) \in W_{\text {loc }}^{2,2}\left(\mathbb{R}^{d}, \mathrm{~d} x\right) ; \nabla V \in L_{\text {loc }}^{4}\left(\mathbb{R}^{d}, \exp (-V) \mathrm{d} x\right)$;

(iii) there exist constants $c, k_{0}>0$ and $\alpha>d$ such that

$$
|V(x)|+|\nabla V(x)|+|\Delta V(x)| \leqslant c(1+|x|)^{-\alpha} \text { for }|x| \geqslant k_{0}
$$

Let $\gamma \in \Gamma_{a d}$. Then $\left(H, C_{0}^{\infty}\left(\mathbb{R}^{d}\right)\right)$ is both $L^{1}$ - and $L^{2}$-unique, i.e., its closure in $L^{1}\left(\mathbb{R}^{d}, \rho_{\gamma} \mathrm{d} x\right)$, $L^{2}\left(\mathbb{R}^{d}, \rho_{\gamma} \mathrm{d} x\right)$ generates a $C_{0}$-semigroup on $L^{1}\left(\mathbb{R}^{d}, \rho_{\gamma} \mathrm{d} x\right), L^{2}\left(\mathbb{R}^{d}, \rho_{\gamma} \mathrm{d} x\right)$, resp.

Furthermore, they constructed a weak solution for (9) under the conditions of Theorem 2.

Lemma 3. Assume that $V$ satisfies the conditions from Theorem 2. Then $\nabla V \in L^{1}\left(\mathbb{R}^{d}, \exp (-V) \mathrm{d} x\right)$. Furthermore, $V$ satisfies the integrability condition

$$
\int_{\mathbb{R}^{d}}\left|\mathrm{e}^{-p V(x)}-1\right| \mathrm{d} x<\infty
$$

for every (inverse temperature) $p \geqslant 1$.

Proof. We have that

$$
\int_{B\left(0, k_{0}\right)^{c}}|\nabla V(x)| \exp (-V(x)) \mathrm{d} x \leqslant \int_{B\left(0, k_{0}\right)^{c}} c(1+|x|)^{-\alpha} \exp \left(c\left(1+\left|k_{0}\right|\right)^{-\alpha}\right) \mathrm{d} x<\infty .
$$

With condition (ii) from Theorem 2 this implies the first assertion.

For the second part let $p \geqslant 1$. Of course,

$$
\int_{B\left(0, k_{0}\right)}\left|\mathrm{e}^{-p V(x)}-1\right| \mathrm{d} x<\infty
$$

Outside $B\left(0, k_{0}\right)$ we have

$$
\begin{aligned}
\int_{B\left(0, k_{0}\right)^{c} \cap\{V \leqslant 0\}}\left|\mathrm{e}^{-p V(x)}-1\right| \mathrm{d} x & =\int_{B\left(0, k_{0}\right)^{c} \cap\{V \leqslant 0\}}\left(\mathrm{e}^{p|V(x)|}-1\right) \mathrm{d} x \\
& \leqslant \int_{B\left(0, k_{0}\right)^{c} \cap\{V \leqslant 0\}}\left(\mathrm{e}^{p c(1+|x|)^{-\alpha}}-1\right) \mathrm{d} x<\infty, \\
\int_{B\left(0, k_{0}\right)^{c} \cap\{V \geqslant 0\}}\left|\mathrm{e}^{-p V(x)}-1\right| \mathrm{d} x & =\int_{B\left(0, k_{0}\right)^{c} \cap\{V \geqslant 0\}}\left(1-\mathrm{e}^{-p V(x)}\right) \mathrm{d} x \\
& \leqslant \int_{B\left(0, k_{0}\right)^{c} \cap\{V \geqslant 0\}}\left(1-\mathrm{e}^{-p c(1-|x|)^{-\alpha}}\right) \mathrm{d} x<\infty .
\end{aligned}
$$

Hence, the assertion follows.

Assume that $V$ satisfies the conditions from Theorem 2. Then the operator $H$ is $L^{2}$-unique or, equivalently, essentially self-adjoint (cf., e.g., [4, Corollary 1.2]). Hence, the corresponding preDirichlet form

$$
\mathcal{E}(f, g)=\int_{\mathbb{R}^{d}}\langle\nabla f, \nabla g\rangle \rho_{\gamma} \mathrm{d} x, \quad f, g \in C_{0}^{\infty}\left(\mathbb{R}^{d}\right),
$$

on $L^{2}\left(\mathbb{R}^{d}, \rho_{\gamma} \mathrm{d} x\right)$ has a unique Dirichlet extension $(\mathcal{E}, D(\mathcal{E}))$. Since we assume that the dimension $d \geqslant 2$, we have that

$$
\left\{x \in \mathbb{R}^{d}: \rho(x)>0\right\}=\mathbb{R}^{d} \backslash \gamma
$$

is connected. Therefore, we can apply [4, Theorem 3.7 (i)] plus a localization argument to obtain the ergodicity of the corresponding semigroup. 


\section{Environment process and invariance principle}

For $x_{0} \in \mathbb{R}^{d}$ define the space shift by $x_{0}$ on $\Gamma$ via

$$
\Theta_{x_{0}} \gamma:=\gamma+x_{0}:=\left\{y+x_{0}: y \in \gamma\right\}, \quad \gamma \in \Gamma .
$$

Then, for a process $X_{t} \in \mathbb{R}^{d} \backslash \gamma, t \geqslant 0$, the corresponding environment process is defined as

$$
\xi_{t}^{\gamma}:=\Theta_{-X_{t}} \gamma, \quad t \geqslant 0
$$

$\xi^{\gamma}$ is a process on $\Gamma$ starting in $\gamma-X_{0}$.

Let $V_{\mathrm{E}}$ ( $E$ stands for environment) be a symmetric, translation invariant potential on $\mathbb{R}^{d}$, and let $\mu_{\mathrm{E}}$ be an extremal grand canonical Gibbs measure on $(\Gamma, \mathcal{B}(\Gamma))$ with respect to $V_{\mathrm{E}}$ and activity parameter $z>0$, which is invariant and ergodic with respect to space shifts $\Theta_{x_{0}}$. We assume, that $\mu_{\mathrm{E}}$ has correlation functions $k_{\mu_{\mathrm{E}}}^{(n)}$ with Ruelle bound $\xi>0$. Furthermore, let $V_{\mathrm{I}}$ satisfy the assumptions of Theorem 1 and Theorem 2 .

The space shift $\Theta_{x_{0}}$ acts on the relative energy via

$$
\Theta_{x_{0}} E_{\mathrm{I}}(x, \gamma):=E_{\mathrm{I}}\left(x, \gamma+x_{0}\right)=\sum_{y \in \gamma} V_{\mathrm{I}}\left(x-\left(y+x_{0}\right)\right)=E_{\mathrm{I}}\left(x-x_{0}, \gamma\right) .
$$

Lemma 4. Let $p \geqslant 1$. Then $\mathrm{e}^{-E_{\mathrm{I}}\left(x_{0}, \cdot\right)} \in L^{p}\left(\Gamma, \mu_{\mathrm{E}}\right)$ for every $x_{0} \in \mathbb{R}^{d}$. Moreover,

$$
\sup _{x_{0} \in \mathbb{R}^{d}}\left\|\mathrm{e}^{-E_{\mathrm{I}}\left(x_{0}, \cdot\right)}\right\|_{L^{p}\left(\Gamma, \mu_{\mathrm{E}}\right)}<\infty
$$

Proof. For $x_{0} \in \mathbb{R}^{d}$ define $\theta:=\theta_{x_{0}}: \mathbb{R}^{d} \rightarrow \mathbb{R}, \theta_{x_{0}}(x):=\left|1-\mathrm{e}^{-p V_{\mathrm{I}}\left(x-x_{0}\right)}\right| \geqslant 0$. Because of the integrability condition (10) for any inverse temperature, in particular for $p$, we have that $\theta_{x_{0}} \in L^{1}\left(\mathbb{R}^{d}, \mathrm{~d} x\right)$. For any $\gamma \in \Gamma$ it holds that

$$
\prod_{y \in \gamma}(1+\theta(y))=1+\sum_{n=1}^{|\gamma|} \sum_{\left\{y_{1}, \ldots, y_{n}\right\} \subset \gamma} \theta\left(y_{1}\right) \cdots \theta\left(y_{n}\right)
$$

Since $f^{(n)}\left(x_{1}, \ldots, x_{n}\right):=\theta\left(x_{1}\right) \cdots \theta\left(x_{n}\right), x_{1}, \ldots, x_{n} \in \mathbb{R}^{d}, n \in \mathbb{N}$, is a non-negative symmetric function on $\left(\mathbb{R}^{d}\right)^{n}$ for any $n$, we have that

$$
\int_{\Gamma} \sum_{\left\{x_{1}, \ldots, x_{n}\right\} \subset \gamma} f^{(n)}\left(x_{1}, \ldots, x_{n}\right) \mathrm{d} \mu_{\mathrm{E}}(\gamma)=\frac{1}{n !} \int_{\left(\mathbb{R}^{d}\right)^{n}} f^{(n)}\left(x_{1}, \ldots, x_{n}\right) k_{\mu_{\mathrm{E}}}^{(n)}\left(x_{1}, \ldots, x_{n}\right) \mathrm{d} x_{1} \cdots \mathrm{d} x_{n}
$$

$n \in \mathbb{N}$. From the Ruelle bound it follows

$$
\begin{aligned}
\int_{\Gamma}\left|\mathrm{e}^{-E_{\mathrm{I}}\left(x_{0}, \gamma\right)}\right|^{p} \mathrm{~d} \mu_{\mathrm{E}}(\gamma) & =\int_{\Gamma} \mathrm{e}^{-p \sum_{y \in \gamma} V_{\mathrm{I}}\left(x_{0}-y\right)} \mathrm{d} \mu_{\mathrm{E}}(\gamma)=\int_{\Gamma} \prod_{y \in \gamma}\left(1+\left(e^{-p V_{\mathrm{I}}\left(x_{0}-y\right)}-1\right)\right) \mathrm{d} \mu_{\mathrm{E}}(\gamma) \\
& \leqslant \int_{\Gamma} \prod_{y \in \gamma}(1+\theta(y)) \mathrm{d} \mu_{\mathrm{E}}(\gamma) \stackrel{(14)}{=} 1+\int_{\Gamma} \sum_{n=1}^{|\gamma|} \sum_{\left\{y_{1}, \ldots, y_{n}\right\} \subset \gamma} \theta\left(y_{1}\right) \cdots \theta\left(y_{n}\right) \mathrm{d} \mu_{\mathrm{E}}(\gamma) \\
& \stackrel{(15)}{\leqslant} 1+\sum_{n=1}^{\infty} \frac{1}{n !} \int_{\left(\mathbb{R}^{d}\right)^{n}} \theta\left(x_{1}\right) \cdots \theta\left(x_{n}\right) k_{\mu_{\mathrm{E}}}^{(n)}\left(x_{1}, \ldots, x_{n}\right) \mathrm{d} x_{1} \cdots \mathrm{d} x_{n} \\
& \stackrel{(3)}{\leqslant} \sum_{n=0}^{\infty} \frac{1}{n !} \xi^{n}\|\theta\|_{L^{1}}^{n}=\mathrm{e}^{\xi\|\theta\|_{L^{1}}}<\infty
\end{aligned}
$$

This proves the first part of the assertion. 
However, the term $C:=\mathrm{e}^{\xi\left\|\theta_{x_{0}}\right\|_{L^{1}}}$ in the last equation is independent of $x_{0}$, since, by translation invariance of the Lebesgue measure, $\left\|\theta_{x_{0}}\right\|_{L^{1}}=\left\|\theta_{0}\right\|_{L^{1}}$ for all $x_{0}$. Thus,

$$
\sup _{x_{0} \in \mathbb{R}^{d}} \int_{\Gamma}\left|\mathrm{e}^{-E_{\mathrm{I}}\left(x_{0}, \gamma\right)}\right|^{p} \mathrm{~d} \mu_{\mathrm{E}}(\gamma) \leqslant C<\infty
$$

Define

$$
\mathrm{d} \mu^{*}(\gamma):=\frac{1}{Z} \mathrm{e}^{-E_{\mathrm{I}}(0, \gamma)} \mathrm{d} \mu_{\mathrm{E}}(\gamma)
$$

where

$$
Z:=\int_{\Gamma} \mathrm{e}^{-E_{\mathrm{I}}(0, \gamma)} \mathrm{d} \mu_{\mathrm{E}}(\gamma)<\infty
$$

by Lemma 4 . Since

$$
\mu_{\mathrm{E}}\left(\left\{\mathrm{e}^{-E_{\mathrm{I}}(0, \cdot)}=0\right\}\right)=\mu_{\mathrm{E}}(\{\gamma: 0 \in \gamma\})=0
$$

we obtain that $\mu_{\mathrm{E}}$ and $\mu^{*}$ are equivalent measures, i.e., they have the same nullsets.

For $\mu_{\mathrm{E}}$-a.e. $\gamma$ we have that $0 \notin \gamma$ and that $\gamma \in \Gamma_{\mathrm{ad}}$. Hence, there exists $\left(X_{t}^{\gamma}\right)_{t \geqslant 0}$, the corresponding solution of (5) with $V=V_{\mathrm{I}}$ and $x_{0}=0$, i.e.,

$$
\left\{\begin{aligned}
\mathrm{d} X_{t}^{\gamma} & =\beta_{\gamma}\left(X_{t}^{\gamma}\right) \mathrm{d} t+\mathrm{d} W_{t}, \\
X_{0}^{\gamma} & =0,
\end{aligned}\right.
$$

with

$$
\beta_{\gamma}=-\sum_{y \in \gamma} \nabla V_{\mathrm{I}}(x-y)
$$

Let $\left(\xi_{t}^{\gamma}\right)_{t \geqslant 0}$ denote the corresponding environment process with start in $\gamma$.

Similarly to $[3$, p. 846$]$ one can prove the following lemma:

Lemma 5. $\left(\xi_{t}\right)_{t \geqslant 0}$ is reversible and ergodic with respect to $\mu^{*}$.

Lemma 6. Let

$$
\Phi(\gamma):=\nabla_{x} E_{\mathrm{I}}(0, \gamma)=\sum_{y \in \gamma} \nabla V_{I}(y)=-\beta_{\gamma}(0)
$$

Then $\Phi \in L^{1}\left(\Gamma, \mu^{*}\right)$.

Proof. Using the Georgii-Ngyuen-Zessin identity (1) we obtain that

$$
\begin{aligned}
\int_{\Gamma}|\Phi(\gamma)| \mathrm{d} \mu^{*}(\gamma) & \leqslant \int_{\Gamma} \sum_{y \in \gamma}\left|\nabla V_{\mathrm{I}}(y)\right| \frac{1}{Z} \mathrm{e}^{-E_{\mathrm{I}}(0, \gamma)} \mathrm{d} \mu_{\mathrm{E}}(\gamma) \\
& =\int_{\Gamma} \int_{\mathbb{R}^{d}}\left|\nabla V_{\mathrm{I}}(y)\right| \frac{1}{Z} \mathrm{e}^{-E_{\mathrm{I}}(0, \gamma \cup y)} \mathrm{e}^{-E_{\mathrm{E}}(y, \gamma)} z \mathrm{~d} y \mathrm{~d} \mu_{\mathrm{E}}(\gamma) \\
& =\frac{z}{Z} \int_{\mathbb{R}^{d}}\left|\nabla V_{\mathrm{I}}(y)\right| \mathrm{e}^{V_{\mathrm{I}}(y)} \int_{\Gamma} \mathrm{e}^{-E_{\mathrm{I}}(0, \gamma)} \mathrm{e}^{-E_{\mathrm{E}}(y, \gamma)} \mathrm{d} \mu_{\mathrm{E}}(\gamma) \mathrm{d} y
\end{aligned}
$$

It follows from Lemma 4 and the Cauchy-Schwarz inequality that

$$
\int_{\Gamma} \mathrm{e}^{-E_{\mathrm{I}}(0, \gamma)} \mathrm{e}^{-E_{\mathrm{E}}(y, \gamma)} \mathrm{d} \mu_{\mathrm{E}}(\gamma)<C, \quad x \in \mathbb{R}^{d},
$$

for some constant $C<\infty$ independent of $x$. Hence, we obtain from the first part of Lemma 3 that

$$
\int_{\Gamma}|\Phi(\gamma)| \mathrm{d} \mu^{*}(\gamma) \leqslant \frac{z C}{Z} \int_{\mathbb{R}^{d}}\left|\nabla V_{\mathrm{I}}(y)\right| \mathrm{e}^{V_{\mathrm{I}}(y)} \mathrm{d} y<\infty .
$$


Rewrite $\left(X_{t}\right)_{t \geqslant 0}$ in terms of the environment process:

$$
X_{t}=-\int_{0} \Phi\left(\xi_{s}\right) \mathrm{d} s+W_{t} .
$$

Then, we can apply [3, Theorem 2.5] to obtain

Theorem 7. For $\varepsilon \rightarrow 0$,

$$
X_{t}^{\varepsilon}:=\varepsilon X_{\varepsilon^{-2} t}
$$

converges (in the sense of finite-dimensional distributions) in $\mu_{\mathrm{E}}$-measure to a Brownian motion on $\mathbb{R}^{d}$.

\section{Acknowledgements}

This article is an overview of my diploma thesis [12]. I want to express my gratitude to my supervisor Prof. Dr. Yuri G. Kondratiev for his support and help with this. Financial support by Deutsche Forschungsgemeinschaft (DFG) via IGK "Stochastics and Real World Models" (Beijing, Bielefeld) is gratefully acknowledged.

\section{References}

1. Albeverio S., Kondratiev Yu.G., Roeckner M., Analysis and Geometry on Configuration Spaces, J. Funct. Anal. 1998, 154, 444-500.

2. Albeverio S., Kondratiev Yu.G., Roeckner M., Analysis and Geometry on Configuration Spaces: The Gibbsian Case, J. Funct. Anal. 1998, 157, 242-291.

3. De Masi A., Ferrari P.A., Goldstein S., Wick W.D., An Invariance Principle for Reversible Markov Processes. Applications to Random Motions in Random Environments, J. Statist. Phys., 1989, 55, Nos. 3/4, 787-855.

4. Eberle A., Uniqueness and Non-Uniqueness of Semigroups Generated by Singular Diffusion Operators, Lecture Notes in Mathematics 1718, Springer, Berlin, 1999.

5. Kipnis C., Varadhan S.R.S., Central Limit Theorem for Additive Functionals of Reversible Markov Processes and Applications to Simple Exclusions, Commun. Math. Phys., 1986, 104, 1-19.

6. Klein A., Yang W.S., A characterization of first order phase transistions for superstable interactions in classical statistical mechanics, J. Statist. Phys., 1993, 71, 1043-1062.

7. Kondratiev Yu.G., Konstantinov A.Yu., Roeckner M., Uniqueness of diffusion generators for two types of particle systems with singular interactions, J. Funct. Anal., 2004, 212, 357-372.

8. Kondratiev Yu.G., Kuna T., Correlation functionals for Gibbs measures and Ruelle bounds, Methods Funct. Anal. Topology, 2003, 9, no. 1, 9-58.

9. Kondratiev Yu.G., Kuna T., Kutoviy O., On relations between a priori bounds for measures on configuration spaces, Infin. Dimens. Anal. Quantum Probab. Relat. Top., 2004, 7, no. 2, 195-213.

10. Krylov N.V., Röckner M., Strong solutions of stochastic equations with singular time dependent drift, Probab. Theory Relat. Fields, 2005, 131, 154-196.

11. Ruelle D., Superstable Interactions in Classical Statistical Mechanics, Commun. Math. Phys., 1970, 18, 127-159.

12. Struckmeier S., Invariance Principle for Diffusions in Random Environment, diploma thesis, Universität Bielefeld, 2005. 


\section{Принцип інваріантності для дифузій у випадковому середовищі}

\section{С.Штрукмайер}

Факультет математики, Університет Білефельда, Німеччина

Отримано 31 січня 2008 р.

Ми показуємо принцип інваріантності для дифузійного руху частинки, що взаємодіє з випадковою нерухомою конфігурацією нескінченною кількістю інших частинок у $\mathrm{IR}^{d}$. Взаємодія описується симетричним, трансляційно інваріантним парним потенціалом з відштовхуванням на нульовій відстані і характерним спаданням на безмежності.

Ключові слова: принцип інваріантності, випадкове середовище, конфігураційний простір, дифузія із сингулярною взаємодією

PACS: $02.50 . \mathrm{Ga}$ 